\title{
Fine structure effective collision strengths for the electron impact excitation of $\mathrm{Al} \mathrm{III}^{\star}$
}

\author{
C. E. Hudson and K. L. Bell
}

\author{
Department of Applied Mathematics and Theoretical Physics, The Queens University of Belfast, Belfast BT7 1NN, \\ Northern Ireland, UK \\ e-mail: c.hudson@am.qub.ac.uk
}

Received 26 October 2004 / Accepted 7 March 2005

\begin{abstract}
A 10-state R-matrix calculation has been carried out to generate fine structure effective collision strengths for the electron impact excitation of doubly ionized aluminium. The target states are represented by configuration interaction wavefunctions and consist of the 10 lowest $L S$ states $-\left(2 p^{6}\right) 3 \mathrm{~s}, 3 \mathrm{p}, \ldots, 5 \mathrm{p}, 5 \mathrm{~d}$. These 10 target states give rise to 17 fine structure levels and 136 possible transitions. The fine structure collision strengths were obtained by transforming to a jj-coupling scheme using the JAJOM program of Saraph and have been determined at a sufficiently fine energy mesh to delineate properly the resonance structure. The effective collision strengths were calculated by averaging the electron collision strengths over a Maxwellian distribution of velocities. Tabulations of the non-zero effective collision strengths for transitions between both the $L S$ states and the fine structure levels are given for electron temperatures $\left(T_{\mathrm{e}}\right)$ in the range $\log _{10} T_{\mathrm{e}}(\mathrm{K})=3.6-5.6$. Comparisons are made with previous calculations and we find good agreement with the work of Kimura et al. (1998).
\end{abstract}

Key words. atomic processes - line: formation - methods: analytical

\section{Introduction}

Astrophysically, recent detections of Al III lines include: the bright Quasi-Stellar Object (QSO) HE 2243-6031, where the spectrum obtained is used to analyse the damped Ly $\alpha$ system (Lopez et al. 2002); the broad absorption line quasar FBQS 0840+3633 (de Kool et al. 2002); the narrow-line quasar I Zwicky 1 (Laor et al. 1997). The lines that have been observed in these objects occur at 1854 and $1862 \AA$ and are attributed to transitions from the $3 \mathrm{p}^{2} \mathrm{P}_{1 / 2,3 / 2}^{\circ}$ levels to the ground state of $3 \mathrm{~s}^{2} \mathrm{~S}$. Lines arising from the $4 \mathrm{~s}^{2} \mathrm{~S}-4 \mathrm{p}^{2} \mathrm{P}^{\circ}$ transition near $5700 \AA$ have also been observed recently in the spectra of HD 77581, the B-supergiant companion of the X-ray pulsar Vela X-1 (Barziv et al. 2001).

The emission lines of the Na-like ions are commonly used to form diagnostics of electron temperature for astrophysical and laboratory plasmas (Flower \& Nussbaumer 1975; Keenan 1993). Hence determinations of excitation rates and effective collision strengths are required. Determinations have been made recently for the absolute emission cross section for the electron-impact excitation of the $3 \mathrm{p}-3 \mathrm{~s}$ transition using crossed beams methods (Dunn et al. 2002) and merged beams methods (Bannister et al. 2002), with the results from the two experiments barely overlapping.

* Table 7 is only available in electronic form at the CDS via anonymous ftp to cdsarc.u-strasbg.fr $(130.79 .128 .5)$ or via http://cdsweb.u-strasbg.fr/cgi-bin/qcat?J/A+A/436/1131
Theoretically, a number of calculations have been carried out in $L S$-coupling to determine the effective collision strengths for the Al III transitions. In this paper we compare with 3 previous calculations, carried out by Kimura et al. (1998), Dufton \& Kingston (1987) and Mitroy \& Norcross (1989).

Kimura et al. (1998) performed an 11-state $R$-matrix calculation using the 11 lowest-lying levels as their target states, and using only real orbitals in their wavefunction expansions. The authors included 30 continuum orbitals and calculated the $R$-matrix radius to be 27.8 au, and considered partial waves for $L=0-10$. Electron excitation collision strengths for transitions between the 7 lowest lying $L S$ states 3s, 3p, 3d, 4s, 4p, 4d, 4f, were calculated and effective collision strengths presented over the electron temperature range $\log _{10} T_{\mathrm{e}}=3.6-5.6$.

The calculation of Dufton \& Kingston (1987) is also an $R$-matrix calculation. The five lowest levels were included as target states, and 25 continuum orbitals were used. Here an $R$-matrix radius of 12 au was determined and partial-wave expansions considered for $L \leq 12$. Effective collision strengths are given for the 10 transitions arising from the transitions between the five lowest $L S$ states for 6 electron temperatures in the range $\log _{10} T_{\mathrm{e}}(\mathrm{K})=4.0-5.5$.

Mitroy \& Norcross (1989) perform a 9-state (5 real states $3 \mathrm{~s}, 3 \mathrm{p}, 3 \mathrm{~d}, 4 \mathrm{~s}, 4 \mathrm{p}$ and 4 pseudo-states $\overline{4 \mathrm{~d}}, \overline{4 \mathrm{f}}, \overline{5 \mathrm{~s}}$, $\overline{5 p})$ close-coupling calculation using semi-empirical HartreeFock wavefunctions, with one- and two-body polarization 
potentials included in the scattering Hamiltonian. The closecoupling equations were solved up to a maximum $L$ value of 12 . An energy mesh $\leq 0.01$ au was used to map out resonance structure and effective collision strengths presented for the four transitions from the ground state to the $3 \mathrm{p}, 3 \mathrm{~d}, 4 \mathrm{~s}$ and $4 p$ levels.

We find that there are discrepancies between the data from these three calculations, which is noted for example by Kimura et al. (1998). The current work aims to resolve the discrepancies between the existing data and provide a set of effective collision strengths that can be confidently applied. We also provide data for a more extensive set of $L S$ transitions than the previous works and give tabulations for the 136 fine structure effective collision strengths between the 17 lowest-lying $J$-resolved levels arising from the 10 lowest-lying $L S$ levels, for electron temperatures in the range $\log _{10} T_{\mathrm{e}}(\mathrm{K})=3.6-5.6$.

\section{Calculation details}

Wavefunctions were constructed for the 10 lowest $L S$ target states of Al III using the configuration interaction code CIV3 (Hibbert 1975). Each target-state wavefunction $\Psi$ is represented by a linear combination of single-configuration functions $\Phi_{i}$, each of which has the same total $L S \pi$ symmetry as the target-state wavefunction

$\Psi(L S)=\sum_{i=1}^{m} a_{i} \Phi_{i}\left(\alpha_{i} L S\right)$

The $\Phi_{i}$ are constructed from a set of one-electron orbitals, with the $\alpha_{i}$ representing the coupling of the angular momenta associated with these one-electron spin orbitals to form the total $L$ and $S$. The mixing coefficients $a_{i}$ are determined by the CIV3 code and are eigenvector components of the Hamiltonian matrix having particular $L S \pi$ symmetry. The Hamiltonian matrix elements are defined as

$H_{i j}=\left\langle\Phi_{i}|H| \Phi_{j}\right\rangle$

where $H$ denotes the Hamiltonian operator. The one-electron orbitals used to construct the $\Phi_{i}$ each consist of a radial function, a spherical harmonic and a spin function:

$u_{n l m_{l} m_{s}}(\mathbf{r}, \sigma)=\frac{1}{r} P_{n l}(r) Y_{l}^{m_{l}}(\theta, \phi) \chi_{m_{s}}(\sigma)$

These orbitals are chosen to be analytic, with the radial part being expressed as a sum of Slater-type orbitals:

$P_{n l}(r)=\sum_{j n l} c_{j n l} r^{I_{j n l}} \exp \left(-\zeta_{j n l} r\right)$

In this expression, for each orbital, the powers of $r$ (the $I_{j n l}$ ) are kept fixed and the coefficients $c_{j n l}$ and exponents $\zeta_{j n l}$ are treated as variational parameters which are optimised by the CIV3 code. For this calculation the ten lowest Al III states were included as the target states, namely $\left(1 \mathrm{~s}^{2} 2 \mathrm{~s}^{2} 2 \mathrm{p}^{6}\right) 3 \mathrm{~s}^{2} \mathrm{~S}, 3 \mathrm{p}^{2} \mathrm{P}^{\circ}$, $3 \mathrm{~d}^{2} \mathrm{D}, 4 \mathrm{~s}^{2} \mathrm{~S}, 4 \mathrm{p}^{2} \mathrm{P}^{\circ}, 4 \mathrm{~d}^{2} \mathrm{D}, 4 \mathrm{f}^{2} \mathrm{~F}^{\circ}, 5 \mathrm{~s}^{2} \mathrm{~S}, 5 \mathrm{p}^{2} \mathrm{P}^{\circ}$ and $5 \mathrm{~d}^{2} \mathrm{D}$. To describe each of these target states $\Psi$ requires the use of thirteen orthogonal one-electron orbitals: $1 \mathrm{~s}, 2 \mathrm{~s}, 2 \mathrm{p}, 3 \mathrm{~s}$, $3 \mathrm{p}, 3 \mathrm{~d}, 4 \mathrm{~s}, 4 \mathrm{p}, 4 \mathrm{~d}, 4 \mathrm{f}, 5 \mathrm{~s}, 5 \mathrm{p}, 5 \mathrm{~d}$. To these we added two pseudo-orbitals $\overline{6 \mathrm{p}}, \overline{6 \mathrm{~d}}$.
Table 1. The Hartree-Fock orbital parameters $(c, I, \zeta)$ of the radial wavefunctions for the $1 \mathrm{~s}, 2 \mathrm{~s}, 3 \mathrm{~s}$ and $2 \mathrm{p}$ orbitals.

\begin{tabular}{|c|c|c|c|}
\hline Orbital & $c_{j n l}$ & $I_{j n l}$ & $\zeta_{j n l}$ \\
\hline \multirow[t]{7}{*}{$1 \mathrm{~s}$} & 0.89975 & 1 & 13.16350 \\
\hline & 0.02450 & 1 & 21.20500 \\
\hline & 0.00511 & 2 & 4.90029 \\
\hline & 0.09175 & 2 & 11.86950 \\
\hline & 0.00091 & 3 & 1.72058 \\
\hline & -0.00062 & 3 & 1.38268 \\
\hline & -0.00156 & 3 & 3.93106 \\
\hline \multirow[t]{7}{*}{$2 \mathrm{~s}$} & -0.23339 & 1 & 13.16350 \\
\hline & -0.00665 & 1 & 21.20500 \\
\hline & 0.87218 & 2 & 4.90029 \\
\hline & -0.11944 & 2 & 11.86950 \\
\hline & -0.00607 & 3 & 1.72058 \\
\hline & 0.00466 & 3 & 1.38268 \\
\hline & 0.25303 & 3 & 3.93106 \\
\hline \multirow[t]{7}{*}{$3 \mathrm{~s}$} & 0.06575 & 1 & 13.16350 \\
\hline & 0.00141 & 1 & 21.20500 \\
\hline & -0.26274 & 2 & 4.90029 \\
\hline & 0.03479 & 2 & 11.86950 \\
\hline & 0.82888 & 3 & 1.72058 \\
\hline & 0.25014 & 3 & 1.38268 \\
\hline & -0.12792 & 3 & 3.93106 \\
\hline \multirow[t]{3}{*}{$2 p$} & 0.63835 & 2 & 3.34979 \\
\hline & 0.38807 & 2 & 5.90995 \\
\hline & 0.03000 & 2 & 11.02820 \\
\hline
\end{tabular}

We used the Hartree-Fock orbital parameters of Clementi \& Roetti (1974) for the 1s, 2s, 2p and 3s orbitals, and these are noted in Table 1. For the remainder of the real orbitals, the CIV3 code was employed to determine the $c_{j n l}, I_{j n l}$ and $\zeta_{\text {jnl }}$ parameters and the values obtained are given in Table 2. For the optimisations of our real orbitals, we note that: the $3 p$ orbital was optimised on the energy of the $2 p^{6} 3 p^{2} \mathrm{P}^{\circ}$ state using this single configuration; the $3 \mathrm{~d}$ was optimised on the $2 \mathrm{p}^{6} 3 \mathrm{~d}^{2} \mathrm{D}$ state also using a single configuration; the $4 \mathrm{~s}$ orbital was optimised on the $2 p^{6} 4 s^{2} S$ level using the $2 p^{6} 3 s$ and $2 p^{6} 4$ s configurations; the $4 p$ was optimised on the energy of the $2 p^{6} 4 p^{2} \mathrm{P}^{\circ}$ level using the $2 p^{6} 3 p$ and $2 p^{6} 4 p$ configurations; the $4 d$ was optimised on the $2 p^{6} 4 d^{2} D$ state with the $2 p^{6} 3 d$ and $2 p^{6} 4 d$ configurations; a $4 f$ orbital was optimised on the $2 \mathrm{p}^{6} 4 \mathrm{f}^{2} \mathrm{~F}^{\circ}$ energy using the single $2 \mathrm{p}^{6} 4 \mathrm{f}$ configuration; the $5 \mathrm{~s}$ orbital was optimised on the $2 \mathrm{p}^{6} 5 \mathrm{~s}^{2} \mathrm{~S}$ state using the $2 p^{6} 3 s, 2 p^{6} 4 s$ and $2 p^{6} 5 s$ configurations; the $5 p$ was optimised on the energy of the $2 p^{6} 5 p^{2} \mathrm{P}^{\circ}$ using the $2 p^{6} 3 p, 2 p^{6} 4 p$ and $2 \mathrm{p}^{6} 5 \mathrm{p}$ configurations.

The pseudo-orbitals were optimised in the following way: a $\overline{6 d}$ orbital was optimised on the energy of the $2 p^{6} 3 p^{2} \mathrm{P}^{\circ}$ level using the $2 \mathrm{p}^{6} 3 \mathrm{p}, 2 \mathrm{p}^{5} 3 \mathrm{~s}^{2}, 2 \mathrm{p}^{5} 3 \mathrm{p}^{2}, 2 \mathrm{p}^{5} n \mathrm{~d}^{2}$ and $2 \mathrm{p}^{5} 3 \mathrm{~s} n \mathrm{~d}(n=3$, $4,5,6)$ configurations, thus allowing for $p \rightarrow d$ correlation; a $\overline{6 p}$ orbital was optimised on the energy of the $2 p^{6} 3 s^{2} S$ state 
Table 2. The optimised $c, I$ and $\zeta$ parameters of the non-Hartree-Fock orbitals.

\begin{tabular}{|c|c|c|c|c|c|c|c|}
\hline Orbital & $c_{j n l}$ & $I_{j n l}$ & $\zeta_{j n l}$ & Orbital & $c_{j n l}$ & $I_{j n l}$ & $\zeta_{j n l}$ \\
\hline \multirow[t]{5}{*}{$3 p$} & 0.03366 & 2 & 8.61695 & $5 \mathrm{~s}$ & 0.03357 & 1 & 10.79734 \\
\hline & 0.22185 & 2 & 4.14929 & & -0.13311 & 2 & 4.12675 \\
\hline & -1.27550 & 3 & 1.19530 & & 0.68984 & 3 & 1.45549 \\
\hline & -0.55129 & 3 & 0.95229 & & -1.94890 & 4 & 0.93519 \\
\hline & 0.86631 & 4 & 1.16488 & & 1.98103 & 5 & 0.75499 \\
\hline \multirow[t]{4}{*}{$3 d$} & 0.01883 & 3 & 4.55109 & $5 p$ & 0.09513 & 2 & 4.80125 \\
\hline & 1.07847 & 3 & 1.02782 & & -1.39634 & 3 & 0.97708 \\
\hline & 0.01832 & 4 & 3.16833 & & 2.79857 & 4 & 0.87847 \\
\hline & -0.09203 & 4 & 1.26339 & & -2.04394 & 5 & 0.68107 \\
\hline \multirow[t]{5}{*}{$4 \mathrm{~s}$} & 0.05138 & 1 & 10.73127 & $5 d$ & 0.01375 & 3 & 4.23472 \\
\hline & -0.20046 & 2 & 4.15522 & & 1.29829 & 3 & 0.84345 \\
\hline & 0.89725 & 3 & 1.49966 & & 0.06945 & 4 & 1.61888 \\
\hline & -1.37119 & 4 & 1.02543 & & -3.47295 & 4 & 0.66642 \\
\hline & & & & & 2.88606 & 5 & 0.59000 \\
\hline \multirow[t]{5}{*}{$4 p$} & 0.08052 & 2 & 6.47210 & & -0.09422 & 5 & 1.91806 \\
\hline & 0.08939 & 3 & 4.32415 & & & & \\
\hline & -0.94849 & 3 & 1.26746 & $\overline{6 p}$ & 2.08071 & 2 & 2.80805 \\
\hline & 1.42295 & 4 & 0.90354 & & -4.45585 & 3 & 2.49277 \\
\hline & & & & & 2.73195 & 4 & 2.15396 \\
\hline \multirow[t]{5}{*}{$4 d$} & 0.01356 & 3 & 4.50464 & & -0.98933 & 5 & 0.84336 \\
\hline & 1.29219 & 3 & 0.98240 & & 0.75385 & 6 & 0.75090 \\
\hline & -1.56655 & 4 & 0.73883 & & & & \\
\hline & -0.19257 & 4 & 1.50582 & $\overline{6 d}$ & 1.04338 & 3 & 5.15462 \\
\hline & & & & & -0.56880 & 4 & 1.74266 \\
\hline \multirow[t]{2}{*}{$4 \mathrm{f}$} & 1.00000 & 4 & 0.75067 & & 0.38948 & 5 & 1.59276 \\
\hline & & & & & -0.03200 & 6 & 0.97942 \\
\hline
\end{tabular}

using the $2 \mathrm{p}^{6} 3 \mathrm{~s}, 2 \mathrm{p}^{5} 3 \mathrm{p} n \mathrm{~d}, 2 \mathrm{p}^{5} n \mathrm{~d} 4 \mathrm{p}, 2 \mathrm{p}^{5} n \mathrm{~d} 5 \mathrm{p}, 2 \mathrm{p}^{5} n \mathrm{~d} \overline{6 \mathrm{p}}$ configurations, which allows for $n \mathrm{p} \rightarrow n$ d correlation. The orbital parameters resulting from these optimisations are also shown in Table 2.

Wavefunctions for the $10 \mathrm{Al}$ III target states were constructed according to Eq. (1) using the orbitals from Tables 1 and 2 to define a set of single-configuration functions generated by a two electron replacement on the $1 s^{2} 2 s^{2} 2 p^{6} 3 s$ basis configuration, with the $1 \mathrm{~s}$ and $2 \mathrm{~s}$ shells being kept closed, at least five electrons in the $2 p$ shell and at most one electron in the 4f shell. This generation leads to a total of 475 configurations $\left(67 \times{ }^{2} \mathrm{~S}, 141 \times{ }^{2} \mathrm{P}^{\circ}, 154 \times{ }^{2} \mathrm{D}\right.$ and $113 \times{ }^{2} \mathrm{~F}^{\circ}$ configurations $)$.

In Table 3, we compare the calculated $L S$ target state energies in Rydbergs $\left(1\right.$ Ryd $\left.=2.17987 \times 10^{-18} \mathrm{~J}\right)$ for Al III relative to the $2 \mathrm{~s}^{2} 2 \mathrm{p}^{6} 3 \mathrm{~s}^{2} \mathrm{~S}$ ground state with experimental values from the NIST database available at http://www.physics.nist.gov. We also tabulate the energy levels calculated by Kimura et al. (1998) and those of Dufton \& Kingston (1987). For each of the calculations we display the energy levels and note the difference of these from the observed values of NIST in parentheses. Such a comparison between the calculated values and the observed data provides a useful test of the wavefunctions. From Table 3 it is clear that the current calculation provides a much better representation of the target states than either of the calculations of Kimura et al. (1998) or Dufton \& Kingston (1987). Dufton \& Kingston (1987) and Kimura et al. (1998) achieve a similar set of targetstate energy levels, although the work of Dufton \& Kingston (1987) only includes the 5 lowest target levels. We find that the current calculation provides energy levels that are to within $1 \%$ of the experimental values, with the exception of the $5 \mathrm{p}$ level which is $1.2 \%$ lower than the experimental value. For the same states, the Kimura et al. data are up to $1.9 \%$ different from the experimental values.

As an additional check on the quality of the wavefunctions for the target-states, we also examine the oscillator strengths produced using our set of wavefunctions. Oscillator strengths for the allowed transitions between the $10 \mathrm{LS}$ target states are given in Table 4 . Comparisons are made in Table 4 with oscillator strengths from several other sources: the frozen-core Hartree-Fock approximation of McEachran \& Cohen (1983) and the calculation of Siegel et al. (1998) which uses the single 
Table 3. Energy levels in Rydbergs for the $10 L S$ target states included in the calculation, relative to the Al III $2 s^{2} 2 p^{6} 3 s^{2} S^{e}$ ground state. Values are shown for the experimental data of NIST along with the values obtained from the current calculation, as well as data from the calculations of Kimura et al. (1998) and Dufton \& Kingston (1987). The differences between the observed values of NIST and the data from each of the three calculations are given in parentheses.

\begin{tabular}{lcccc}
\hline \hline & \multicolumn{3}{c}{ Energy in Rydbergs } \\
\cline { 2 - 5 }$L S$ state & NIST & Current & Kimura et al. & Dufton \& Kingston \\
\hline $2 \mathrm{p}^{6} 3 \mathrm{~s}^{2} \mathrm{~S}^{\mathrm{e}}$ & 0.0000 & $0.0000(0.0000)$ & $0.0000(0.0000)$ & $0.0000(0.0000)$ \\
$2 \mathrm{p}^{6} 3 \mathrm{p}^{2} \mathrm{P}^{\circ}$ & 0.4906 & $0.4848(0.0058)$ & $0.4857(0.0049)$ & $0.4860(0.0046)$ \\
$2 \mathrm{p}^{6} 3 \mathrm{~d}^{2} \mathrm{D}^{\mathrm{e}}$ & 1.0567 & $1.0490(0.0077)$ & $1.0397(0.0170)$ & $1.0374(0.0193)$ \\
$2 \mathrm{p}^{6} 4 \mathrm{~s}^{2} \mathrm{~S}^{\mathrm{e}}$ & 1.1497 & $1.1391(0.0106)$ & $1.1288(0.0209)$ & $1.1288(0.0209)$ \\
$2 \mathrm{p}^{6} 4 \mathrm{p}^{2} \mathrm{P}^{\circ}$ & 1.3094 & $1.2977(0.0117)$ & $1.2875(0.0219)$ & $1.2888(0.0206)$ \\
$2 \mathrm{p}^{6} 4 \mathrm{~d}^{2} \mathrm{D}^{\mathrm{e}}$ & 1.5108 & $1.4990(0.0118)$ & $1.4880(0.0228)$ & \\
$2 \mathrm{p}^{6} 4 \mathrm{f}^{2} \mathrm{~F}^{\circ}$ & 1.5274 & $1.5130(0.0144)$ & $1.4991(0.0283)$ & \\
$2 \mathrm{p} 65 \mathrm{~s}^{2} \mathrm{~S}^{\mathrm{e}}$ & 1.5550 & $1.5418(0.0132)$ & $1.5293(0.0257)$ & \\
$2 \mathrm{p}^{6} 5 \mathrm{p}^{2} \mathrm{P}^{\circ}$ & 1.6262 & $1.6136(0.0126)$ & $1.6000(0.0262)$ & \\
$2 \mathrm{p} \mathrm{p}^{6} 5 \mathrm{~d}^{2} \mathrm{D}^{\mathrm{e}}$ & 1.7212 & $1.7078(0.0134)$ & $1.6949(0.0263)$ & \\
\hline
\end{tabular}

Table 4. Oscillator strengths for the allowed transitions between the $10 L S$ target states included in the current calculation. Values are given for the length and velocity forms of the oscillator strength $\left(f_{l}\right.$ and $\left.f_{v}\right)$ and are compared, where available, to the works of: M \& C - McEachran \& Cohen (1983); S et al. - Siegel et al. (1998); D \& K - Dufton \& Kingston (1987); K et al. - Kimura et al. (1998).

\begin{tabular}{|c|c|c|c|c|c|c|c|c|c|}
\hline \multirow[b]{2}{*}{ Transition } & \multicolumn{2}{|c|}{ Current } & \multicolumn{2}{|c|}{$M \& C$} & \multirow{2}{*}{$\frac{\mathrm{S} \text { et al. }}{f_{l}}$} & \multicolumn{2}{|c|}{ D \& K } & \multicolumn{2}{|c|}{$\mathrm{K}$ et al. } \\
\hline & $f_{l}$ & $f_{v}$ & $f_{l}$ & $f_{v}$ & & $f_{l}$ & $f_{v}$ & $f_{l}$ & $f_{v}$ \\
\hline $2 p^{6} 3 s^{2} S-2 p^{6} 3 p^{2} P^{\circ}$ & 0.8564 & 0.8614 & 0.869 & 0.859 & 0.8350 & 0.890 & 0.847 & 0.8889 & 0.8442 \\
\hline$-2 p^{6} 4 p^{2} \mathrm{P}^{\circ}$ & 0.0135 & 0.0137 & 0.0133 & 0.0134 & 0.0151 & 0.0108 & 0.0116 & 0.0129 & 0.0125 \\
\hline$-2 p^{6} 5 p^{2} \mathrm{P}^{\circ}$ & 0.0060 & 0.0070 & 0.0076 & 0.0076 & 0.0087 & & & & \\
\hline $2 p^{6} 4 s^{2} S-2 p^{6} 4 p^{2} P^{\circ}$ & 1.2895 & 1.2700 & 1.28 & 1.27 & 1.277 & 1.303 & 1.286 & & \\
\hline$-2 p^{6} 5 p^{2} P^{\circ}$ & 0.0036 & 0.0050 & 0.0062 & 0.0063 & 0.0059 & & & & \\
\hline $2 p^{6} 5 s^{2} S-2 p^{6} 5 p^{2} P^{\circ}$ & 1.7167 & 1.6629 & 1.66 & 1.66 & 1.674 & & & & \\
\hline $2 p^{6} 3 p^{2} P^{\circ}-2 p^{6} 4 s^{2} S$ & 0.1301 & 0.1302 & 0.127 & 0.126 & 0.1313 & 0.132 & 0.127 & & \\
\hline$-2 p^{6} 5 s^{2} S$ & 0.0184 & 0.0183 & 0.0182 & 0.0178 & 0.0190 & & & & \\
\hline $2 p^{6} 4 p^{2} P^{\circ}-2 p^{6} 5 s^{2} S$ & 0.2326 & 0.2301 & 0.229 & 0.229 & 0.2332 & & & & \\
\hline $2 p^{6} 3 p^{2} P^{\circ}-2 p^{6} 3 d^{2} D$ & 0.9154 & 0.9149 & 0.921 & 0.935 & 0.8975 & 0.928 & 0.966 & & \\
\hline$-2 p^{6} 4 d^{2} D$ & 0.0038 & 0.0035 & 0.0036 & 0.0042 & 0.0027 & & & & \\
\hline$-2 p^{6} 5 d^{2} D$ & $2 \times 10^{-6}$ & 0.0001 & 0.0002 & 0.0001 & 0.0000 & & & & \\
\hline $2 p^{6} 4 p^{2} P^{\circ}-2 p^{6} 4 d^{2} D$ & 1.2855 & 1.2832 & 1.28 & 1.29 & 1.276 & & & & \\
\hline$-2 p^{6} 5 d^{2} D$ & 0.0244 & 0.0227 & 0.0225 & 0.0233 & 0.0214 & & & & \\
\hline $2 p^{6} 5 p^{2} P^{\circ}-2 p^{6} 5 d^{2} D$ & 1.6191 & 1.5748 & 1.60 & 1.61 & 1.598 & & & & \\
\hline $2 p^{6} 3 d^{2} D-2 p^{6} 4 p^{2} P^{\circ}$ & 0.1719 & 0.1715 & 0.162 & 0.170 & 0.1705 & & & & \\
\hline$-2 p^{6} 5 p^{2} \mathrm{P}^{\circ}$ & 0.0095 & 0.0092 & 0.0096 & 0.0099 & 0.0099 & & & & \\
\hline $2 p^{6} 4 d^{2} D-2 p^{6} 5 p^{2} P^{\circ}$ & 0.3459 & 0.3342 & 0.343 & 0.347 & 0.3439 & & & & \\
\hline $2 p^{6} 3 d^{2} D-2 p^{6} 4 f^{2} F^{\circ}$ & 0.9471 & 0.9455 & 0.937 & 0.935 & & & & & \\
\hline $2 p^{6} 4 d^{2} D-2 p^{6} 4 f^{2} F^{\circ}$ & 0.0810 & 0.0842 & 0.100 & 0.102 & & & & & \\
\hline $2 p^{6} 4 f^{2} F^{\circ}-2 p^{6} 5 d^{2} D$ & 0.0166 & 0.0170 & 0.0187 & 0.0189 & & & & & \\
\hline
\end{tabular}

configuration Dirac-Fock method; as well as the oscillator strengths given by the 5 -state $R$-matrix calculation of Dufton \& Kingston (1987) and the values noted by Kimura et al. (1998) from their 11-state calculation. We look at how our values compare to these other data and also at the agreement between the length and velocity forms of the oscillator strengths. 
For the 21 transitions noted in Table 4, we find that the length and velocity agreement achieved for the current calculation is as good as that observed in the work of McEachran \& Cohen (1983), except in a few cases - namely those where the oscillator strength is very small. Excluding the four smallest oscillator strengths in Table 4 ( $3 s-5 p, 4 s-5 p, 3 p-4 d$ and $3 p-5 d)$ means that for the remaining 17 transitions, the current calculation achieves agreement in the length and velocity forms of the oscillator strengths to within $7 \%$. Considering the same transitions, the McEachran and Cohen length and velocity forms of the oscillator strengths agree to within 5\%. We achieve better length and velocity agreement than the values given by Dufton $\&$ Kingston (1987) for 4 out of 5 of their transitions, the exception being the $2 p^{6} 4 s-2 p^{6} 4 p$ transition. Here, the Dufton \& Kingston (1987) length and velocity values lie slightly closer together being 1.303 and 1.286 respectively, compared to the current calculation values of $f_{l}=1.2895$ and $f_{v}=1.2700$. We also find that the current calculation achieves better length and velocity agreement for the two transitions given in Kimura et al. (1998).

When we consider a comparison of the length forms of the oscillator strengths between the different calculations noted in Table 4, we find that the present work agrees with the data of McEachran \& Cohen (1983) to within 8\% when we ignore three of the smallest oscillator strengths $(3 \mathrm{~s}-5 \mathrm{p}, 4 \mathrm{~s}-5 \mathrm{p}$ and $3 \mathrm{p}-5 \mathrm{~d})$ and the $4 \mathrm{~d}-4 \mathrm{f}$ and $4 \mathrm{f}-5 \mathrm{~d}$ transitions. For the 18 transitions of Siegel et al. (1998), ignoring four of the weaker transitions ( $3 s-5 p, 4 s-5 p, 3 p-4 d$ and $3 p-5 d)$, we find there is agreement to within $12 \%$ for the length forms when compared to the current data. For the Dufton \& Kingston (1987) values, the length forms of the oscillator strengths for these 5 transitions differ by only a few per cent except for the $3 \mathrm{~s}-4 \mathrm{p}$ transition which differs by $20 \%$ from the current value. We also find that the two transitions quoted in Kimura et al. (1998), i.e. 3s-3p and $3 \mathrm{~s}-4 \mathrm{p}$, are both within $4 \%$ of the current work. Therefore, overall, we find that for the oscillator strengths, the current work has the best agreement with the work of McEachran \& Cohen (1983).

All of this analysis of the energy levels and oscillator strengths generated using the current set of target state wavefunctions leads us to be confident that we have an accurate representation of the target system. Using these target state wavefunctions, the $(N+1)$ "ion-plus-electron" system is described using the $R$-matrix method outlined by Burke \& Robb (1975), using the associated computer codes detailed by Berrington et al. (1987). Configurations describing the $(N+1)$-electron symmetries were generated through the addition of one electron from the orbital set to those configurations generated above by the two-electron replacement on the basis configuration. The $R$-matrix radius was calculated to be 28.2 atomic units and for each orbital angular momentum, 35 Schmidtorthogonalized continuum orbitals were included, ensuring that a converged collision strength was obtained up to an incident electron energy of 7.95 Rydbergs $\left(1 \mathrm{Ryd}=2.17987 \times 10^{-18} \mathrm{~J}\right)$. The correct positioning of resonances relative to the target states included in the calculation was ensured by adjusting our theoretical energies to the experimental values of NIST, prior to diagonalization of the Hamiltonian matrix.
In forming the $(N+1)$ symmetries, we consider all total angular momenta $0 \leq L \leq 12$. Adding an electron to the target state symmetries, which are all doublets, means that for the $(N+1)$ symmetries we now include both even and odd parities for singlet and triplet multiplicities. For dipole-allowed transitions, however, it is necessary to consider the effect of higher partial waves with $L>12$ because they have a significant effect upon the collision strengths. The contributions from these higher partial waves is approximated by assuming the partial collision strengths form a geometric series expansion, with a geometric scaling factor given by the ratio of two adjacent terms. This "topping-up" procedure has been used successfully in similar calculations of Bell \& Ramsbottom (2000), and Ramsbottom et al. (2001).

For small energy intervals between fine structure levels, it is possible to recouple the matrices obtained from non-relativistic calculations in $L S$ coupling to obtain the electron collision strengths between these levels. Above all the thresholds the effects of intermediate coupling in the target can be included by using this method. In the current work, the program of Saraph (1978) is used to carry out this recoupling procedure. Further details of the coupling scheme are given by Bell \& Ramsbottom (2000).

Effective collision strengths $\Upsilon_{i f}$ for a particular electron temperature $T_{\mathrm{e}}$ (in Kelvin) were obtained by averaging the electron collision strengths $\Omega_{i f}$ over a Maxwellian distribution of velocities, so that

$\Upsilon_{i \mathrm{f}}\left(T_{\mathrm{e}}\right)=\int_{0}^{\infty} \Omega_{i \mathrm{f}}\left(E_{\mathrm{f}}\right) \exp \left(-E_{\mathrm{f}} / k T_{\mathrm{e}}\right) \mathrm{d}\left(E_{\mathrm{f}} / k T_{\mathrm{e}}\right)$

where $E_{\mathrm{f}}$ is the final free electron energy after excitation and $k$ is Boltzmann's constant.

\section{Results and discussion}

The collision strengths calculated in this work have been evaluated for a fine mesh of incident impact energies, at energy intervals of 0.0004 Rydbergs across the energy range considered ( 0 to $8 \mathrm{Ryd}$ ). This ensured that the autoionizing resonances which converge to the target state thresholds were fully delineated.

Those resonances located at energies lower than the highest target threshold considered in this work $\left(2 p^{6} 5 d^{2} D\right)$ are physically meaningful; however at higher energies (i.e. above 1.7212 Ryd) pseudo-resonances appear. These arise from the inclusion of pseudo-orbitals in the wavefunction expansion (Burke et al. 1981). At higher temperatures the high-impact energy region is much more important and so it is necessary to properly average over the pseudo-resonances to prevent distortion of the correct results in the calculation of the effective collision strengths. Thus above the last target state energy, the pseudo-resonances are smoothed over using a cubic spline fit.

The inclusion of the $10 \mathrm{LS}$ target states leads to $17 \mathrm{~J}$-levels and a total of 136 transitions. In analysing the fine structure effective collision strengths we find that the ratio predicted by the statistical weights applies to within $20 \%$. Therefore we only consider the effective collision strengths for the transitions between the $L S$ states which are the sum of the fine structure 
Table 5. Comparison of the current effective collision strengths with those from Kimura et al. (1998) at a selection of electron temperatures in the range $\log _{10} T_{\mathrm{e}}=3.6-5.6(\mathrm{~K})$. The values from Kimura et al. are displayed in brackets alongside the data from the current work.

\begin{tabular}{|c|c|c|c|c|c|c|}
\hline \multirow[b]{2}{*}{ Transition } & \multicolumn{6}{|c|}{$\log _{10} T_{\mathrm{e}}(\mathrm{K})$} \\
\hline & 3.6 & 4.0 & 4.4 & 4.8 & 5.2 & 5.6 \\
\hline $3 s-3 p$ & $1.50^{1}\left(1.49^{1}\right)$ & $1.57^{1}\left(1.60^{1}\right)$ & $1.69^{1}\left(1.74^{1}\right)$ & $1.86^{1}\left(1.94^{1}\right)$ & $2.22^{1}\left(2.34^{1}\right)$ & $3.02^{1}\left(3.13^{1}\right)$ \\
\hline $3 d$ & $3.35(2.65)$ & $3.17(2.89)$ & $3.02(2.93)$ & $2.97(2.95)$ & $3.12(3.21)$ & $3.27(3.70)$ \\
\hline $4 \mathrm{~s}$ & $1.11(1.01)$ & $1.07(1.02)$ & $9.75^{-1}\left(9.66^{-1}\right)$ & $9.06^{-1}\left(9.08^{-1}\right)$ & $9.15^{-1}\left(9.28^{-1}\right)$ & $9.76^{-1}(1.01)$ \\
\hline $4 p$ & $8.17^{-1}\left(7.81^{-1}\right)$ & $7.65^{-1}\left(7.63^{-1}\right)$ & $6.79^{-1}\left(6.91^{-1}\right)$ & $5.72^{-1}\left(5.95^{-1}\right)$ & $5.07^{-1}\left(5.43^{-1}\right)$ & $4.76^{-1}\left(5.26^{-1}\right)$ \\
\hline $4 d$ & $6.50^{-1}\left(6.09^{-1}\right)$ & $5.44^{-1}\left(5.19^{-1}\right)$ & $4.79^{-1}\left(4.57^{-1}\right)$ & $4.52^{-1}\left(4.28^{-1}\right)$ & $4.42^{-1}\left(4.25^{-1}\right)$ & $4.37^{-1}\left(4.31^{-1}\right)$ \\
\hline $4 \mathrm{f}$ & $4.85^{-1}\left(4.63^{-1}\right)$ & $4.75^{-1}\left(4.43^{-1}\right)$ & $4.69^{-1}\left(4.29^{-1}\right)$ & $4.68^{-1}\left(4.32^{-1}\right)$ & $4.53^{-1}\left(4.46^{-1}\right)$ & $4.05^{-1}\left(4.45^{-1}\right)$ \\
\hline $3 p-3 d$ & $3.25^{1}\left(2.66^{1}\right)$ & $3.33^{1}\left(3.07^{1}\right)$ & $3.47^{1}\left(3.37^{1}\right)$ & $3.81^{1}\left(3.81^{1}\right)$ & $4.78^{1}\left(4.86^{1}\right)$ & $6.59^{1}\left(6.88^{1}\right)$ \\
\hline $4 \mathrm{~s}$ & $3.70(2.98)$ & $3.38(2.99)$ & $3.05(2.85)$ & $2.91(2.80)$ & $3.39(3.36)$ & $4.58(5.06)$ \\
\hline $4 p$ & $6.34(6.13)$ & $6.11(6.06)$ & $5.64(5.67)$ & $5.14(5.22)$ & $5.05(5.20)$ & $5.23(5.60)$ \\
\hline $4 d$ & $3.95(3.79)$ & $3.49(3.36)$ & $3.17(3.03)$ & $3.04(2.91)$ & 3.08 (2.97) & $3.29(3.12)$ \\
\hline $4 \mathrm{f}$ & $4.21(3.80)$ & $4.13(3.92)$ & $4.17(4.21)$ & $4.31(4.05)$ & $4.34(4.33)$ & $3.96(4.86)$ \\
\hline $3 d-4 s$ & $1.03^{1}(9.36)$ & $1.11^{1}\left(1.07^{1}\right)$ & $1.00^{1}\left(1.03^{1}\right)$ & $8.08(8.90)$ & $6.71(7.58)$ & $5.71(7.61)$ \\
\hline $4 p$ & $3.82^{1}\left(4.29^{1}\right)$ & $3.89^{1}\left(4.07^{1}\right)$ & $3.72^{1}\left(4.07^{1}\right)$ & $3.66^{1}\left(3.91^{1}\right)$ & $4.29^{1}\left(4.13^{1}\right)$ & $5.54^{1}\left(5.75^{1}\right)$ \\
\hline $4 d$ & $3.33^{1}\left(3.28^{1}\right)$ & $2.69^{1}\left(2.65^{1}\right)$ & $2.30^{1}\left(2.35^{1}\right)$ & $2.12^{1}\left(2.18^{1}\right)$ & $2.05^{1}\left(1.98^{1}\right)$ & $2.01^{1}\left(2.05^{1}\right)$ \\
\hline $4 \mathrm{f}$ & $4.92^{1}\left(4.33^{1}\right)$ & $5.18^{1}\left(4.59^{1}\right)$ & $5.73^{1}\left(5.73^{1}\right)$ & $6.82^{1}\left(7.00^{1}\right)$ & $8.63^{1}\left(8.37^{1}\right)$ & $1.09^{2}\left(1.25^{2}\right)$ \\
\hline $4 s-4 p$ & $4.63^{1}\left(4.62^{1}\right)$ & $5.04^{1}\left(5.10^{1}\right)$ & $5.56^{1}\left(6.71^{1}\right)$ & $6.85^{1}\left(8.13^{1}\right)$ & $9.80^{1}\left(9.79^{1}\right)$ & $1.40^{2}\left(1.47^{2}\right)$ \\
\hline $4 d$ & $1.23^{1}\left(1.13^{1}\right)$ & $1.12^{1}\left(1.25^{1}\right)$ & $1.12^{1}\left(1.64^{1}\right)$ & $1.15^{1}\left(1.38^{1}\right)$ & $1.09^{1}\left(1.23^{1}\right)$ & $8.80\left(1.35^{1}\right)$ \\
\hline $4 f$ & $8.53(7.91)$ & $8.15(9.40)$ & $7.88\left(1.20^{1}\right)$ & $7.55(9.57)$ & $6.79(7.47)$ & $5.44(6.73)$ \\
\hline $4 p-4 d$ & $7.76^{1}\left(7.09^{1}\right)$ & $7.87^{1}\left(9.59^{1}\right)$ & $8.97^{1}\left(1.51^{2}\right)$ & $1.19^{2}\left(1.35^{2}\right)$ & $1.67^{2}\left(1.57^{2}\right)$ & $2.16^{2}\left(2.66^{2}\right)$ \\
\hline $4 \mathrm{f}$ & $3.35^{1}\left(3.38^{1}\right)$ & $3.26^{1}\left(4.30^{1}\right)$ & $3.24^{1}\left(5.95^{1}\right)$ & $3.33^{1}\left(4.82^{1}\right)$ & $3.30^{1}\left(4.09^{1}\right)$ & $2.92^{1}\left(4.60^{1}\right)$ \\
\hline $4 d-4 f$ & $3.44^{2}\left(6.58^{2}\right)$ & $3.45^{2}\left(9.97^{2}\right)$ & $5.00^{2}\left(1.57^{3}\right)$ & $8.95^{2}\left(1.29^{3}\right)$ & $1.56^{3}\left(9.72^{2}\right)$ & $2.21^{3}\left(9.67^{2}\right)$ \\
\hline
\end{tabular}

data, but the data for all 136 fine structure transitions are available at CDS. In Table 5 we make comparison with the effective collision strengths given in Kimura et al. (1998) for all of the transitions between the $3 \mathrm{~s}, 3 \mathrm{p}, 3 \mathrm{~d}, 4 \mathrm{~s}, 4 \mathrm{p}, 4 \mathrm{~d}$ and $4 \mathrm{f} L S$ levels that these authors present. In Table 6 we present the current effective collision strengths for the 45 transitions between the $10 L S$ target states used in this calculation, at a range of electron temperatures $\left(\log _{10} T_{\mathrm{e}}(\mathrm{K})=3.6-5.6\right)$ suitable for application in plasma and astronomical diagnostics. Table 7, available at $\mathrm{CDS}$, gives the fine structure effective collision strength data and contains the following information: Col. 1 lists the transitions between fine-structure states indicated as initial-final according to the J-index assigned to each fine-structure levels noted in the accompanying table. For example, 1-14 denotes the transition $2 s^{2} 2 p^{6} 3 s^{2} S_{1 / 2}-2 s^{2} 2 p^{6} 5 p^{2} P_{1 / 2}^{\circ}$. The remaining columns list the effective collision strengths for each transition at $\log$ arithmic electron temperatures $\log _{10} T_{\mathrm{e}}(\mathrm{K})=3.6-5.6$ in steps of 0.1 dex.

We make some general comments on the comparison in Table 5 after we examine a few specific cases. In Figs. 1-3 we consider in more detail three transitions of particular interest: the $3 s-3 p$, and $4 s-4 p$ transitions that have been noted earlier as recent astrophysical observations and also the
$3 s-4 p$ transition which is explicitly discussed by Kimura et al. (1998). For these three transitions we present both the electron collision strengths and the maxwellian averaged effective collision strengths and make comparison with the works of Kimura et al. (1998), Dufton \& Kingston (1987) and Mitroy $\&$ Norcross (1989), where these data are available.

The upper plot of Fig. 1 displays the effective collision strengths for the $3 \mathrm{~s}-3 \mathrm{p}$ transition from the current work along with those from the calculations of Kimura et al. (1998), Dufton \& Kingston (1987) and Mitroy \& Norcross (1989). The lower plot gives the present electron collision strength for the excitation from the ground state $2 \mathrm{p}^{6} 3 \mathrm{~s}^{2} \mathrm{~S}_{1 / 2}$ level to the $2 \mathrm{p}^{6} 3 \mathrm{p}^{2} \mathrm{P}_{1 / 2}^{\circ}$ level. Attention is drawn to the near-threshold resonance which is also a feature of other transitions (see Figs. 2 and 3). Such features impact significantly on the calculation of the low-temperature effective collision strength. We have used a sufficiently small energy mesh to ensure accuracy in the calculation of the low-temperature effective collision strengths. For this transition, we find that there is good convergence between the four calculations considered. The largest discrepancy exists between the Dufton \& Kingston (1987) work and that of Mitroy \& Norcross (1989), where in particular, at $\log _{10} T_{\mathrm{e}}(\mathrm{K})=5.2$ a difference in the region of $15 \%$ occurs. 
Table 6. Effective Collision Strengths for transitions between the 10 lowest $L S$ levels of $\mathrm{Al}$ III, for electron temperatures ranging from $\log _{10} T_{\mathrm{e}}(\mathrm{K})=3.6$ to 5.6 in steps of 0.2 dex. A superscript indicates the power of 10 with which the number must be multiplied i.e., $a^{-n}=a \times 10^{-n}$.

\begin{tabular}{|c|c|c|c|c|c|c|c|c|c|c|c|}
\hline \multirow[b]{2}{*}{ Transition } & \multicolumn{11}{|c|}{$\log _{10} T_{\mathrm{e}}(\mathrm{K})$} \\
\hline & 3.6 & 3.8 & 4.0 & 4.2 & 4.4 & 4.6 & 4.8 & 5.0 & 5.2 & 5.4 & 5.6 \\
\hline $3 s-3 p$ & $1.50^{1}$ & $1.53^{1}$ & $1.57^{1}$ & $1.63^{1}$ & $1.69^{1}$ & $1.77^{1}$ & $1.86^{1}$ & $2.00^{1}$ & $2.22^{1}$ & $2.56^{1}$ & $3.02^{1}$ \\
\hline$-3 d$ & 3.35 & 3.26 & 3.17 & 3.09 & 3.02 & 2.97 & 2.97 & 3.03 & 3.12 & 3.22 & 3.27 \\
\hline$-4 s$ & 1.11 & 1.10 & 1.07 & 1.02 & $9.75^{-1}$ & $9.32^{-1}$ & $9.06^{-1}$ & $9.00^{-1}$ & $9.15^{-1}$ & $9.44^{-1}$ & $9.76^{-1}$ \\
\hline$-4 p$ & $8.17^{-1}$ & $7.88^{-1}$ & $7.65^{-1}$ & $7.30^{-1}$ & $6.79^{-1}$ & $6.22^{-1}$ & $5.72^{-1}$ & $5.34^{-1}$ & $5.07^{-1}$ & $4.88^{-1}$ & $4.76^{-1}$ \\
\hline$-4 d$ & $6.50^{-1}$ & $5.91^{-1}$ & $5.44^{-1}$ & $5.06^{-1}$ & $4.79^{-1}$ & $4.62^{-1}$ & $4.52^{-1}$ & $4.47^{-1}$ & $4.42^{-1}$ & $4.37^{-1}$ & $4.37^{-1}$ \\
\hline$-4 \mathrm{f}$ & $4.85^{-1}$ & $4.80^{-1}$ & $4.75^{-1}$ & $4.71^{-1}$ & $4.69^{-1}$ & $4.69^{-1}$ & $4.68^{-1}$ & $4.63^{-1}$ & $4.53^{-1}$ & $4.34^{-1}$ & $4.05^{-1}$ \\
\hline$-5 \mathrm{~s}$ & $3.05^{-1}$ & $3.01^{-1}$ & $2.94^{-1}$ & $2.86^{-1}$ & $2.77^{-1}$ & $2.68^{-1}$ & $2.60^{-1}$ & $2.54^{-1}$ & $2.47^{-1}$ & $2.42^{-1}$ & $2.38^{-1}$ \\
\hline$-5 p$ & $2.66^{-1}$ & $2.59^{-1}$ & $2.48^{-1}$ & $2.34^{-1}$ & $2.20^{-1}$ & $2.07^{-1}$ & $1.95^{-1}$ & $1.84^{-1}$ & $1.72^{-1}$ & $1.60^{-1}$ & $1.49^{-1}$ \\
\hline$-5 d$ & $2.13^{-1}$ & $2.14^{-1}$ & $2.15^{-1}$ & $2.17^{-1}$ & $2.18^{-1}$ & $2.19^{-1}$ & $2.17^{-1}$ & $2.11^{-1}$ & $2.02^{-1}$ & $1.90^{-1}$ & $1.77^{-1}$ \\
\hline $3 p-3 d$ & $3.25^{1}$ & $3.28^{1}$ & $3.33^{1}$ & $3.39^{1}$ & $3.47^{1}$ & $3.59^{1}$ & $3.81^{1}$ & $4.19^{1}$ & $4.78^{1}$ & $5.59^{1}$ & $6.59^{1}$ \\
\hline$-4 s$ & 3.70 & 3.53 & 3.38 & 3.21 & 3.05 & 2.93 & 2.91 & 3.05 & 3.39 & 3.93 & 4.58 \\
\hline$-4 p$ & 6.34 & 6.21 & 6.11 & 5.92 & 5.64 & 5.35 & 5.14 & 5.05 & 5.05 & 5.12 & 5.23 \\
\hline$-4 d$ & 3.95 & 3.71 & 3.49 & 3.31 & 3.17 & 3.08 & 3.04 & 3.05 & 3.08 & 3.14 & 3.29 \\
\hline$-4 f$ & 4.21 & 4.15 & 4.13 & 4.13 & 4.17 & 4.24 & 4.31 & 4.36 & 4.34 & 4.21 & 3.96 \\
\hline$-5 \mathrm{~s}$ & $8.63^{-1}$ & $8.42^{-1}$ & $8.09^{-1}$ & $7.67^{-1}$ & $7.21^{-1}$ & $6.79^{-1}$ & $6.45^{-1}$ & $6.24^{-1}$ & $6.22^{-1}$ & $6.45^{-1}$ & $6.91^{-1}$ \\
\hline$-5 p$ & 1.74 & 1.70 & 1.64 & 1.57 & 1.49 & 1.42 & 1.36 & 1.29 & 1.24 & 1.19 & 1.16 \\
\hline$-5 d$ & 1.22 & 1.22 & 1.24 & 1.25 & 1.26 & 1.26 & 1.24 & 1.20 & 1.15 & 1.09 & 1.04 \\
\hline $3 d-4 s$ & $1.03^{1}$ & $1.09^{1}$ & $1.11^{1}$ & $1.07^{1}$ & $1.00^{1}$ & 9.04 & 8.08 & 7.30 & 6.71 & 6.22 & 5.71 \\
\hline$-4 p$ & $3.82^{1}$ & $3.88^{1}$ & $3.89^{1}$ & $3.83^{1}$ & $3.72^{1}$ & $3.62^{1}$ & $3.66^{1}$ & $3.87^{1}$ & $4.29^{1}$ & $4.89^{1}$ & $5.54^{1}$ \\
\hline$-4 d$ & $3.33^{1}$ & $2.97^{1}$ & $2.69^{1}$ & $2.46^{1}$ & $2.30^{1}$ & $2.19^{1}$ & $2.12^{1}$ & $2.08^{1}$ & $2.05^{1}$ & $2.03^{1}$ & $2.01^{1}$ \\
\hline$-4 f$ & $4.92^{1}$ & $5.02^{1}$ & $5.18^{1}$ & $5.41^{1}$ & $5.73^{1}$ & $6.19^{1}$ & $6.82^{1}$ & $7.63^{1}$ & $8.63^{1}$ & $9.78^{1}$ & $1.09^{2}$ \\
\hline$-5 \mathrm{~s}$ & 4.12 & 4.02 & 3.82 & 3.53 & 3.19 & 2.82 & 2.44 & 2.06 & 1.71 & 1.39 & 1.10 \\
\hline$-5 p$ & 6.31 & 6.03 & 5.67 & 5.25 & 4.83 & 4.46 & 4.15 & 3.93 & 3.82 & 3.86 & 4.06 \\
\hline$-5 \mathrm{~d}$ & 6.20 & 6.23 & 6.27 & 6.30 & 6.32 & 6.30 & 6.21 & 6.06 & 5.85 & 5.59 & 5.28 \\
\hline $4 s-4 p$ & $4.63^{1}$ & $4.82^{1}$ & $5.04^{1}$ & $5.27^{1}$ & $5.56^{1}$ & $6.04^{1}$ & $6.85^{1}$ & $8.10^{1}$ & $9.80^{1}$ & $1.19^{2}$ & $1.40^{2}$ \\
\hline$-4 d$ & $1.23^{1}$ & $1.17^{1}$ & $1.12^{1}$ & $1.11^{1}$ & $1.12^{1}$ & $1.14^{1}$ & $1.15^{1}$ & $1.14^{1}$ & $1.09^{1}$ & 9.94 & 8.80 \\
\hline$-4 f$ & 8.53 & 8.33 & 8.15 & 8.00 & 7.88 & 7.74 & 7.55 & 7.24 & 6.79 & 6.18 & 5.44 \\
\hline$-5 \mathrm{~s}$ & 4.36 & 4.35 & 4.30 & 4.23 & 4.18 & 4.17 & 4.20 & 4.27 & 4.37 & 4.45 & 4.49 \\
\hline$-5 p$ & 2.55 & 2.50 & 2.42 & 2.32 & 2.24 & 2.18 & 2.17 & 2.21 & 2.35 & 2.62 & 2.96 \\
\hline$-5 \mathrm{~d}$ & 1.84 & 1.87 & 1.92 & 1.98 & 2.05 & 2.12 & 2.16 & 2.15 & 2.08 & 1.93 & 1.73 \\
\hline $4 p-4 d$ & $7.76^{1}$ & $7.74^{1}$ & $7.87^{1}$ & $8.24^{1}$ & $8.97^{1}$ & $1.02^{2}$ & $1.19^{2}$ & $1.41^{2}$ & $1.67^{2}$ & $1.92^{2}$ & $2.16^{2}$ \\
\hline$-4 f$ & $3.35^{1}$ & $3.30^{1}$ & $3.26^{1}$ & $3.23^{1}$ & $3.24^{1}$ & $3.28^{1}$ & $3.33^{1}$ & $3.35^{1}$ & $3.30^{1}$ & $3.15^{1}$ & $2.92^{1}$ \\
\hline$-5 \mathrm{~s}$ & $1.04^{1}$ & $1.04^{1}$ & $1.03^{1}$ & $1.02^{1}$ & $1.04^{1}$ & $1.11^{1}$ & $1.24^{1}$ & $1.43^{1}$ & $1.63^{1}$ & $1.79^{1}$ & $1.86^{1}$ \\
\hline$-5 p$ & $1.95^{1}$ & $1.93^{1}$ & $1.91^{1}$ & $1.88^{1}$ & $1.85^{1}$ & $1.85^{1}$ & $1.85^{1}$ & $1.86^{1}$ & $1.86^{1}$ & $1.85^{1}$ & $1.82^{1}$ \\
\hline$-5 \mathrm{~d}$ & $3.44^{2}$ & $3.36^{2}$ & $3.45^{2}$ & $3.95^{2}$ & $5.00^{2}$ & $6.65^{2}$ & $8.95^{2}$ & $1.20^{3}$ & $1.56^{3}$ & $1.92^{3}$ & $2.21^{3}$ \\
\hline $4 d-4 f$ & $3.44^{2}$ & $3.36^{2}$ & $3.45^{2}$ & $3.95^{2}$ & $5.00^{2}$ & $6.65^{2}$ & $8.95^{2}$ & $1.20^{3}$ & $1.56^{3}$ & $1.92^{3}$ & $2.21^{3}$ \\
\hline$-5 \mathrm{~s}$ & $2.80^{1}$ & $2.80^{1}$ & $2.73^{1}$ & $2.63^{1}$ & $2.55^{1}$ & $2.51^{1}$ & $2.50^{1}$ & $2.45^{1}$ & $2.34^{1}$ & $2.15^{1}$ & $1.91^{1}$ \\
\hline$-5 p$ & $8.38^{1}$ & $8.62^{1}$ & $8.93^{1}$ & $9.42^{1}$ & $1.02^{2}$ & $1.14^{2}$ & $1.31^{2}$ & $1.50^{2}$ & $1.66^{2}$ & $1.74^{2}$ & $1.72^{2}$ \\
\hline$-5 d$ & $5.40^{1}$ & $5.47^{1}$ & $5.56^{1}$ & $5.68^{1}$ & $5.82^{1}$ & $5.95^{1}$ & $6.03^{1}$ & $6.05^{1}$ & $5.96^{1}$ & $5.77^{1}$ & $5.46^{1}$ \\
\hline $4 f-5 s$ & $1.63^{1}$ & $1.57^{1}$ & $1.46^{1}$ & $1.31^{1}$ & $1.15^{1}$ & 9.89 & 8.39 & 7.02 & 5.78 & 4.65 & 3.64 \\
\hline$-5 p$ & $4.13^{1}$ & $4.05^{1}$ & $3.89^{1}$ & $3.68^{1}$ & $3.43^{1}$ & $3.15^{1}$ & $2.84^{1}$ & $2.50^{1}$ & $2.14^{1}$ & $1.79^{1}$ & $1.48^{1}$ \\
\hline$-5 \mathrm{~d}$ & $5.40^{1}$ & $5.47^{1}$ & $5.56^{1}$ & $5.68^{1}$ & $5.82^{1}$ & $5.95^{1}$ & $6.03^{1}$ & $6.05^{1}$ & $5.96^{1}$ & $5.77^{1}$ & $5.46^{1}$ \\
\hline $5 s-5 p$ & $8.74^{1}$ & $9.15^{1}$ & $9.74^{1}$ & $1.06^{2}$ & $1.20^{2}$ & $1.41^{2}$ & $1.69^{2}$ & $2.02^{2}$ & $2.32^{2}$ & $2.53^{2}$ & $2.59^{2}$ \\
\hline$-5 \mathrm{~d}$ & $3.52^{1}$ & $3.54^{1}$ & $3.55^{1}$ & $3.52^{1}$ & $3.42^{1}$ & $3.21^{1}$ & $2.90^{1}$ & $2.52^{1}$ & $2.11^{1}$ & $1.72^{1}$ & $1.38^{1}$ \\
\hline $5 p-5 d$ & $1.86^{2}$ & $1.95^{2}$ & $2.09^{2}$ & $2.28^{2}$ & $2.53^{2}$ & $2.83^{2}$ & $3.15^{2}$ & $3.42^{2}$ & $3.59^{2}$ & $3.61^{2}$ & $3.49^{2}$ \\
\hline
\end{tabular}



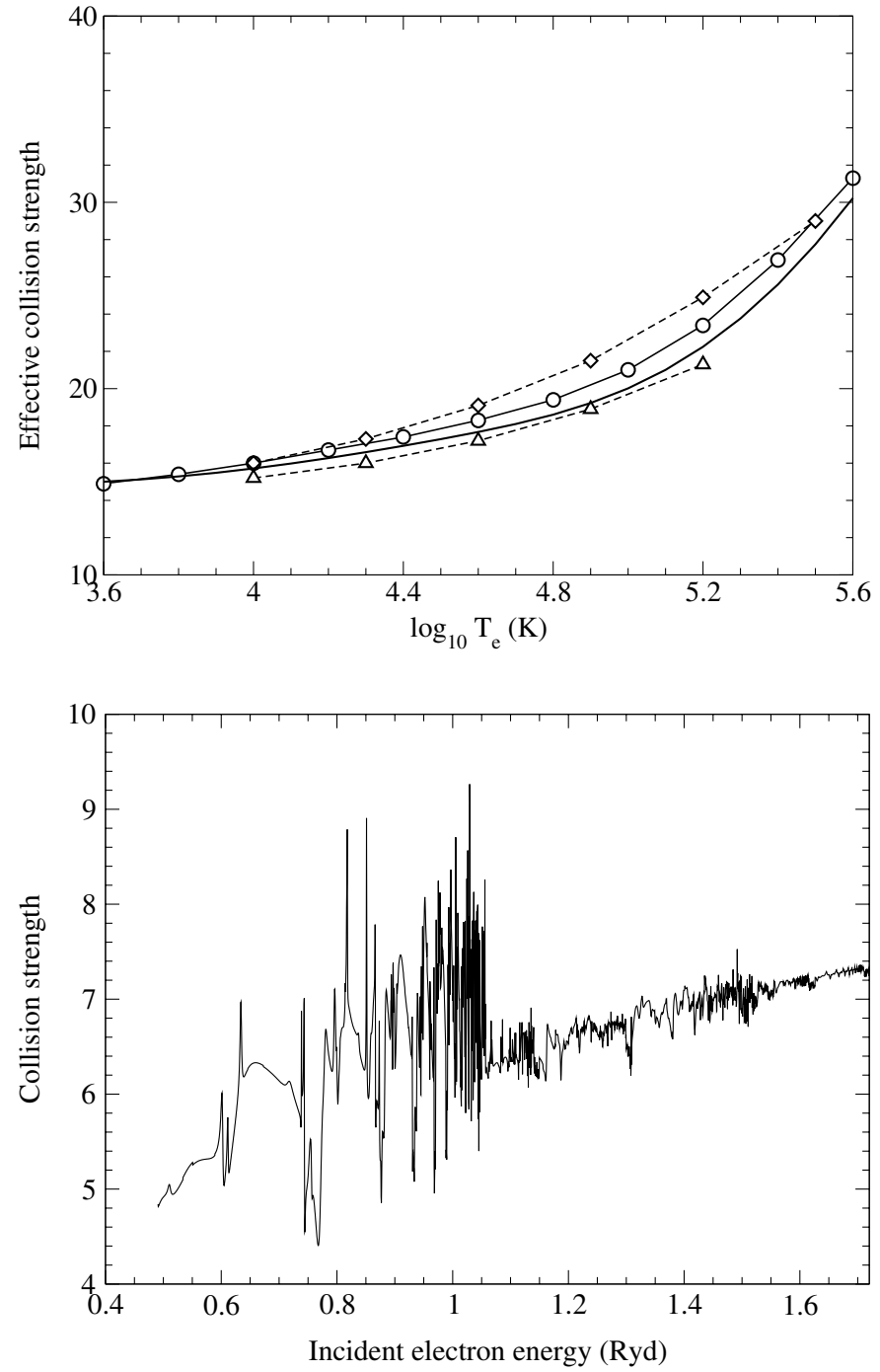

Fig. 1. Upper graph - effective collision strengths for the $3 \mathrm{~s}-3 \mathrm{p}$ excitation for $\mathrm{Al}$ III as a function of the electron temperature (in K): - present, $\bigcirc$ Kimura et al. (1998), $\diamond$ Dufton \& Kingston (1987), $\triangle$ Mitroy \& Norcross (1989). Lower graph - electron collision strengths for one of the contributing fine structure transitions, namely, $3 \mathrm{~s}^{2} \mathrm{~S}_{1 / 2}-3 \mathrm{p}^{2} \mathrm{P}_{1 / 2}^{\circ}$. The $3 \mathrm{~s}^{2} \mathrm{~S}_{1 / 2}-3 \mathrm{p}^{2} \mathrm{P}_{3 / 2}^{\circ}$ collision strength is a factor of two times the data shown, as predicted by the statistical weights.

The current calculation is in closest agreement with the effective collision strength of Mitroy \& Norcross and differs from the Kimura et al. data by less than $5 \%$.

Figure 2 shows the electron collision strength for the $2 p^{6} 3 s^{2} S_{1 / 2}-2 p^{6} 4 p^{2} P_{1 / 2}^{\circ}$ excitation in the lower portion, and in the upper part comparison is made between the current effective collision strength and the previous calculations. It is clear that the current work agrees well with the data of Kimura et al. (1998), with differences of only a few per cent being found for most of the temperature range, although a difference in the region of $10 \%$ is found at the high temperature extremity. We find much more severe discrepancies with the data of Dufton \& Kingston (1987) and Mitroy \& Norcross. The Dufton \& Kingston (1987) data are $20 \%$ lower than the present results at low temperatures and $27 \%$ higher at high temperatures, whilst the Mitroy \& Norcross (1989) effective collision strength is
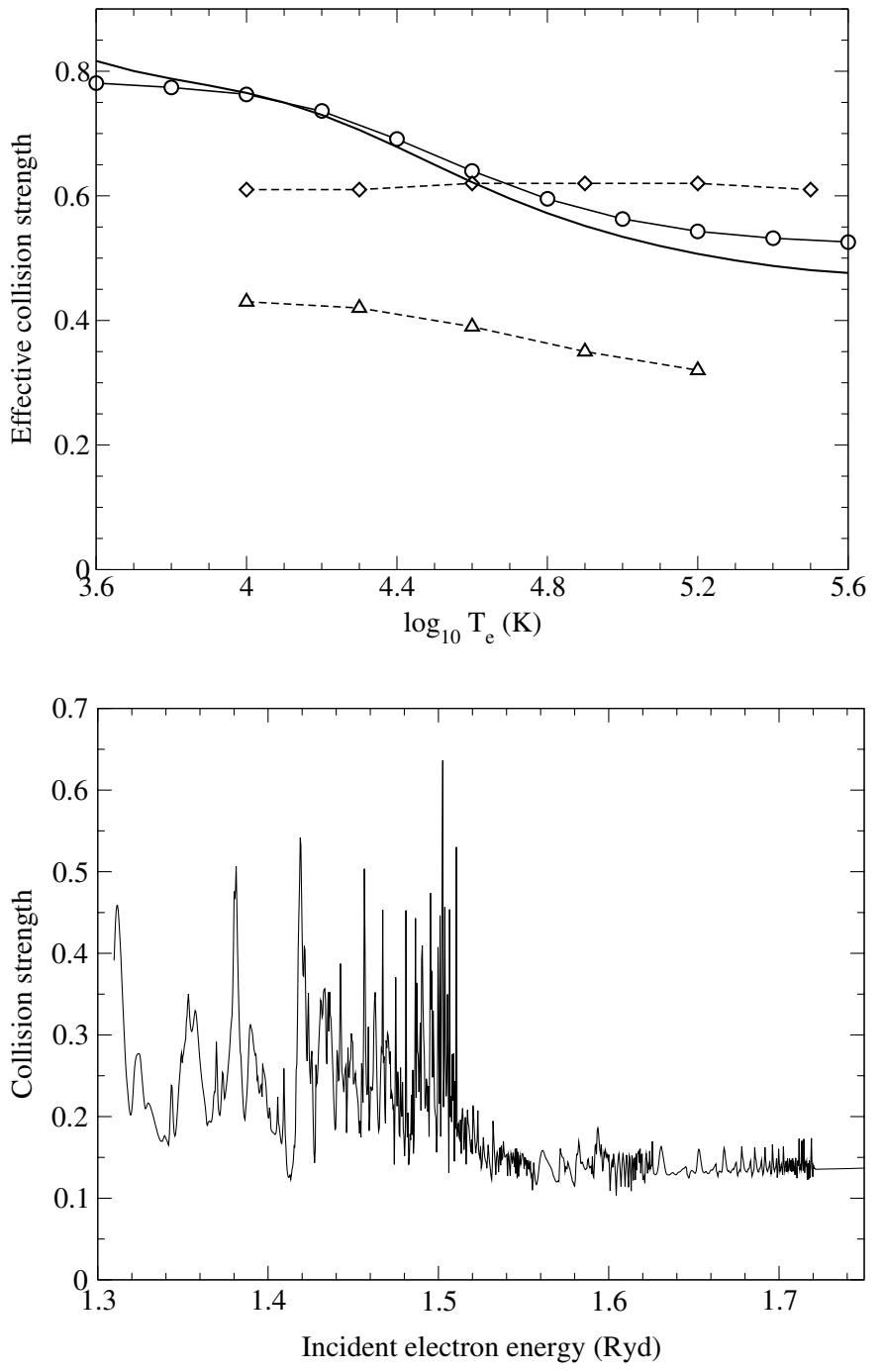

Fig. 2. Upper graph - effective collision strengths for the $3 s-4 p$ excitation for $\mathrm{Al} \mathrm{III}$ as a function of the electron temperature (in K): - present, $\bigcirc$ Kimura et al. (1998), $\diamond$ Dufton \& Kingston (1987), $\triangle$ Mitroy \& Norcross (1989). Lower graph - electron collision strength for one of the two contributing fine structure transitions, namely, $3 \mathrm{~s}^{2} \mathrm{~S}_{1 / 2}-4 \mathrm{p}^{2} \mathrm{P}_{1 / 2}^{\circ}$. The $3 \mathrm{~s}^{2} \mathrm{~S}_{1 / 2}-4 \mathrm{p}^{2} \mathrm{P}_{3 / 2}^{\circ}$ collision strength is a factor of two times this data.

lower than the present calculated values, over the entire range of temperatures, by $30-40 \%$.

In Fig. 3, we show the effective collision strength for the $4 \mathrm{~s}-4 \mathrm{p} L S$ transition, along with the collision strength for the $2 p^{6} 4 s^{2} S_{1 / 2}-2 p^{6} 4 p^{2} P_{1 / 2}^{\circ}$ fine structure transition. Here we agree well with the Kimura et al. (1998) data both at the high temperature and the low temperature extremity, but we have a different shape of effective collision strength in the mid-temperature range. The Kimura et al. (1998) data have a definite "bump" in the curve, centred at $\log _{10} T_{\mathrm{e}}(\mathrm{K})=4.6$, which doesn't seem to be apparent in the work of Dufton \& Kingston (1987), although the effective collision strength of Dufton \& Kingston (1987) is larger than the current work at this point and does coincide with the Kimura et al. (1998) data in this region.

We further note from Table 5, that for all the excitations from the $n=4$ levels, the data of Kimura et al. (1998) 

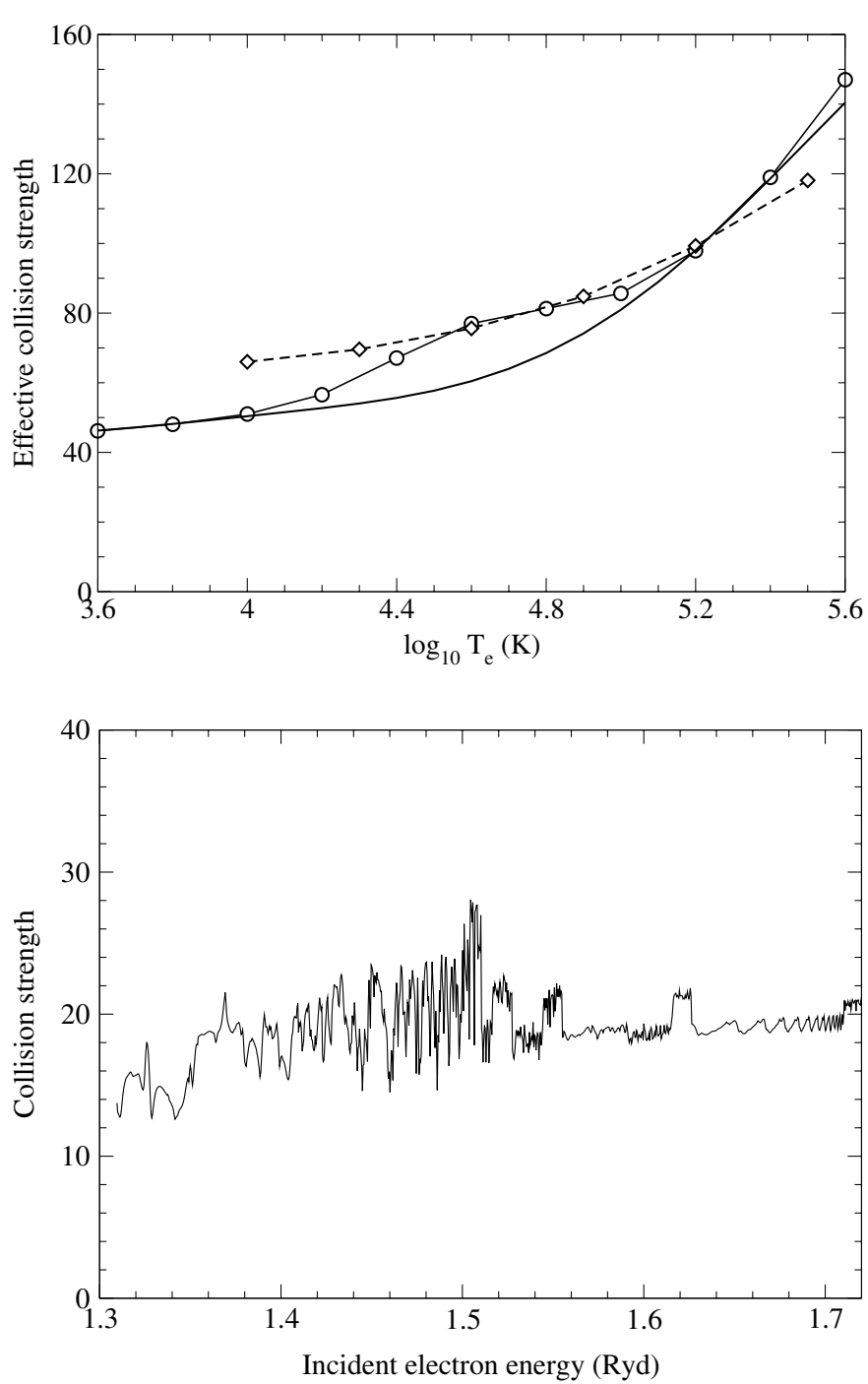

Fig. 3. Upper graph - effective collision strengths for the $4 s-4 p$ excitation for $\mathrm{AlIII}$ as a function of the electron temperature (in K): - present, $\bigcirc$ Kimura et al. (1998), $\diamond$ Dufton \& Kingston (1987). Lower graph - electron collision strength for one of the two contributing fine structure transitions, namely, $4 \mathrm{~s}^{2} \mathrm{~S}_{1 / 2}-4 \mathrm{p}^{2} \mathrm{P}_{1 / 2}^{\circ}$. The $4 \mathrm{~s}^{2} \mathrm{~S}_{1 / 2}-4 \mathrm{p}^{2} \mathrm{P}_{3 / 2}^{\circ}$ collision strength is a factor of two times this data.

exhibits a similar "bump" in the same temperature region, and that agreement with the current calculation exists only at the lower temperatures and briefly around $\log _{10} T_{\mathrm{e}}(\mathrm{K})=5.0$, if at all. For excitations from the $n=3$ levels, we find better agreement with the data of Kimura et al. (1998), than with the results of Dufton \& Kingston (1987) and Mitroy \& Norcross (1989). The majority of effective collision strengths agree with the Kimura et al. (1998) data to within 10\%, apart from some instances at the high or low temperature extremities.

\section{Conclusions}

In this paper we present effective collision strengths for the electron impact excitation of the Al III ion. These astrophysically important atomic data are evaluated for the electron temperature range $\log _{10} T_{\mathrm{e}}(\mathrm{K})=3.6-5.6$ and for all transitions among the lowest $10 \mathrm{LS}$ states of Al III, corresponding to 17 fine structure levels and 136 individual fine structure transitions. We have compared our results to three other calculations, among which there were discrepancies, and in general we find good agreement with Kimura et al. (1998).

The present calculation is certainly the most sophisticated so far. The target state representations, and consequently the $(N+1)$ "ion-plus-electron" system representations, judged by the target state energies and oscillator strengths, are highly accurate and so the present effective collision strengths should also be of high accuracy.

All the effective collision strength data for the $L S$ and fine structure transitions over the temperature range $\log _{10} T_{\mathrm{e}}(\mathrm{K})=$ 3.6-5.6 (in steps of $0.1 \mathrm{dex}$ ) are available by contacting the authors. The electron collision strengths are also available.

Acknowledgements. This work has been supported by PPARC, under the auspices of a Rolling Grant. The calculations were carried out on the ENIGMA supercomputer at the HiPerSPACE Computing Centre, UCL, which is funded by the UK Particle Physics and Astronomy Research Council.

\section{References}

Bannister, M. E., Krause, H. F., Djurić, N., et al. 2002, Phys. Rev. A, 66,032707

Barziv, O., Kaper, L., Van Kerkwijk, M. H., Telting, J. H., \& Van Paradijs, J. 2001, A\&A, 377, 925

Bell, K. L., \& Ramsbottom, C. A. 2000, Atomic Data \& Nuclear Data Tables 76, 176

Berrington, K. A., Burke, P. G., Butler, K., et al. 1987, J. Phys. B, 20, 6379

Burke, P. G., \& Robb, W. D. 1975 Adv. At. Mol. Phys., 11, 143

Burke, P. G., Sukamar, C. V., \& Berrington, K. A. 1981, J. Phys. B, 14,289

Clementi, E., \& Roetti, C. 1974, Atomic Data \& Nuclear Data Tables, 14,177

de Kool, M., Becker, R. B., Arav, N., Gregg, M. D., \& White, R. L. 2002, ApJ, 570, 514

Dufton, P. L., \& Kingston, A. E. 1987, J. Phys. B, 20, 3899

Dunn, G. H., Belić, D. S., \& Cisneros, C. 2002, Phys. Rev. A, 66, 032706

Flower, D. R., \& Nussbaumer, H. 1975, A\&A, 42, 265

Hibbert, A. 1975, Comput. Phys. Comm., 9, 141

Keenan, F. P. 1993, in UV and X-ray Spectroscopy of Laboratory and Astrophysical Plasmas, ed. E. H. Silver, \& S. M. Kahn (Cambridge: Cambridge University Press), 44

Kimura, E., Ohsaki, A., Nakazaki, S., \& Itikawa, Y. 1998, A\&AS, 132, 99

Laor, A., Jannuzi, B. T., Green, R. F., \& Boroson, T. A. 1997, ApJ, 489, 656

Lopez, S., Reimers, D., D’Odorico, S., \& Prochaska, J. X. 2002, A\&A, 385, 778

McEachran, R. P., \& Cohen, M. 1983, J. Phys. B, 16, 3125

Mitroy, J., \& Norcross, D. W. 1989, Phys. Rev. A, 39, 537

Ramsbottom, C. A., Bell, K. L., \& Keenan, F. P. 2001, Atomic Data \& Nuclear Data Tables, 77, 57

Saraph, H. E. 1978, Comput. Phys. Comm., 15, 247

Siegel, W., \& Migdalek, J. 1998, Atomic Data \& Nuclear Data Tables, 68,303 